\title{
On the Condensation of Interstellar Material
}

\author{
E. Schatzman \\ Institut d'Astrophysique, Paris, France
}

$\mathrm{O}$ NE seeks the condition of spontaneous collapse of a layer of interstellar matter by the method of marginal stability. The radiation field is included in this treatment of the gas-dynamic properties of the interstellar material. The optical model used is that of Ambartsumian's, which neglects interlocking.

\section{DESCRIPTION OF THE PROBLEM}

We study the hydrodynamical properties of interstellar material, including the effect of the radiation field. Any motion of the medium modifies the density of the material and, as a result, the radiation field. This last modification reacts in turn on the ionization equilibrium and, in consequence, on the motion of the material. One sees intuitively that, if the optical thickness of the medium in the Lyman continuum is sufficiently great, an increase in the density may be accompanied by a large increase in the number of neutral hydrogen atoms and, in consequence, by a decrease in pressure. Thus an irreversible contraction of the medium is produced which may ultimately lead to a spontaneous transformation of an HII region into an HI region.

The equations describing the problem are:

$$
\begin{gathered}
\text { Ionization } \\
\frac{\partial n_{\mathrm{H}}}{\partial t}=q n_{e} n_{p}-\frac{\sigma}{h \nu} S n_{\mathrm{H}},
\end{gathered}
$$

where $q n_{e} n_{p}$ is the number of recombinations per second, $\sigma$ the photoelectric ionization cross section at the Lyman limit, $S$ the mean total intensity of the ionizing radiation, and $N$ the total number of hydrogen atoms.

\section{Radiative Transfer}

We consider a slab of interstellar material of thickness $2 l$, of optical thickness at the Lyman limit $2 \tau_{1}$. That slab of interstellar material is illuminated from both sides by a radiation field of flux $2 F$, normal to the surface.

Introducing the ratio $\tilde{\omega}$ of the number of re-emissions in the Lyman continuum to the total number of reemissions, we follow Chandrasekhar ${ }^{1}$ in writing the transfer equations

$$
\frac{\cos \theta}{\sigma n_{\mathrm{H}}}\left(\frac{1}{c} \frac{\partial I}{\partial t}+\frac{\partial I}{\partial x}\right)=-I+\varpi \bar{I}+\varpi F e^{-r_{1}} \cosh \tau .
$$

${ }^{1}$ S. Chandrasekhar, Radiative Transfer (Oxford University Press, New York, 1950).
The mean total intensity, which in this problem is the source function, is

$$
S=\bar{I}+F e^{-\tau_{1}} \cosh \tau .
$$

\section{Equation of Wave Motion}

The pressure is

$$
P=\left(2 N-n_{\mathrm{H}}\right) k T .
$$

Thus, the equation for propagation of small disturbances is

$$
\frac{\partial^{2} N}{\partial t^{2}}=\frac{\partial^{2}}{\partial x^{2}} \frac{k}{m_{0}} T\left(2 N-n_{\mathrm{H}}\right)
$$

Solving simultaneous Eqs. (1), (2), and (4) would be difficult. Consequently, by considering various relaxation times, we ask which perturbations can be treated as independent of each other.

(a) According to Spitzer and Savedoff, ${ }^{2}$ relaxation time of the electron temperature in an HII region at $5000^{\circ} \mathrm{K}$ is $1.4 \times 10^{4}$ years $/ n_{p}$, where $n_{p}$ is protons $\mathrm{cm}^{-3}$. Also, temperature changes very little with density. Consequently, we suppose the temperature constant.

(b) The time of restoration of equilibrium ionization after a change in the neutral hydrogen density can be calculated from Eq. (1). Assuming no change in $S$, conditions near equilibrium, and $n_{\mathrm{H}} \ll N$, we compute a relaxation time $h \nu / \sigma S$ for a perturbation in $n_{\mathrm{H}}$ which, for $T_{r}=2 \times 10^{4}$, a dilution factor of $10^{-14}$, and $\sigma=6 \times 10^{-18}$ gives $\sim 1$ year relaxation time. Thus, we assume ionization equilibrium.

(c) For simplicity neglect re-emission in Eq. (2), $\varpi=0$. Then the equation permits a solution, for a perturbation in $I$ holding $n_{\mathrm{H}}$ constant, of the form $g(c t-x) \exp (-t / \cos \theta)$. Thus a perturbation of the radiation field propagates with the velocity of light. Since the dimensions of interstellar clouds are $\sim 1 \mathrm{pc}$, thus relaxation $\sim 1 \mathrm{yr}$, we assume radiative equilibrium.

\section{SOLUTION FOR A SMALL DISTURBANCE}

Equation (1) with left side zero leads to the following relations between $\delta N, \delta n_{\mathrm{H}}$ and $\delta S$ :

$$
\delta N=\frac{N+n_{\mathrm{H}}}{2 n_{\mathrm{H}}} \delta n_{\mathrm{H}}+\frac{N-n_{\mathrm{H}}}{2} \frac{\delta S}{S} .
$$

\footnotetext{
${ }^{2}$ L. Spitzer and M. Savedoff, Astrophys. J. 111, 593 (1950).
} 
From Eq. (2) we can derive the source function, using Chandrasekhar's first approximation,

$$
\begin{aligned}
S= & \frac{2}{2-3 \varpi} F e^{-r_{1}} \\
& \times\left\{\cosh \tau-\frac{3 \varpi}{2} \frac{\cosh \tau_{1}+3^{-\frac{1}{2}} \sinh \tau_{1}}{\cosh p \tau_{1}+p 3^{-\frac{1}{2}} \sinh p \tau_{1}} \cosh p \tau\right\},
\end{aligned}
$$

where $p^{2}=3(1-\varpi)$. We consider a perturbation $\delta N(x) \exp \lambda t$. Equation (5) becomes

$$
\lambda^{2} \delta N(x)-\frac{k}{m_{0}} T \frac{\partial^{2}}{\partial x^{2}}\left(2 \delta N-\delta n_{\mathrm{H}}\right)=0 .
$$

The method of marginal stability consists in finding the solution of $\mathrm{Eq}$. (8) for $\lambda=0$, satisfying the boundary conditions.

TABLE I.

\begin{tabular}{cccc}
\hline \multicolumn{1}{c}{$\boldsymbol{\beta}$} & $T$ & $\boldsymbol{\sigma}$ & $\boldsymbol{p}$ \\
\hline 0.5 & 316000 & 0.660 & 1.01 \\
1 & 158000 & 0.625 & 1.06 \\
2 & 79000 & 0.573 & 1.13 \\
5 & 31600 & 0.505 & 1.22 \\
10 & 15800 & 0.454 & 1.28 \\
20 & 7900 & 0.404 & 1.34 \\
30 & 3160 & 0.347 & 1.40 \\
\hline
\end{tabular}

We therefore consider the equation

$$
\left(\partial^{2} / \partial x^{2}\right)\left[2 \delta N-\delta n_{\mathrm{H}}\right]=0,
$$

and we arbitrarily set $\delta N=0$ at the boundaries of the slab of interstellar matter $(x= \pm l)$. Any other boundary condition would give a similar result.

In order to solve Eq. (9), we have to introduce a variation of the source function $S$, calculated from Eq. (7), into Eq. (6) and to carry it into Eq. (9). The complexity of Eq. (7) makes the algebra somewhat lengthy. However, because the optical thickness $\tau_{1}$ is always large, the problem turns out simpler than it first appears, and a good approximation to the eigenvalue can be obtained.

We limit outselves to a quotation of the results. We introduce a new quantity $n_{0}$, the number of neutral hydrogen atoms in a radiation field of dilution $W$,

$$
n_{0}=N^{2} W^{-1} \cdot 10^{-18.44} \text {. }
$$

The optical depth $\tau_{1}$ is related to $n_{0}$ by the relation

$$
\begin{aligned}
\frac{n_{0}}{N}=\frac{2}{2-3 \varpi}\left\{\left[1+\frac{3 \varpi}{2} \frac{\sqrt{3}-1}{p(\sqrt{3}+p)}\right] e^{-2 \tau_{1}}\right. & \\
& -3 \varpi \frac{\sqrt{3}(\sqrt{3}+1)}{(\sqrt{3}+p)^{2}} e^{-2 p \tau_{1}} .
\end{aligned}
$$

TABLE II.

\begin{tabular}{lrl}
\hline \hline$\varpi$ & $\lambda(\mathrm{pc})$ & $\log \mu$ \\
\hline 0 & 675 & 7.92 \\
0.39 & 1000 & 8.44 \\
0.66 & 1600 & 9.04 \\
\hline
\end{tabular}

To the optical thickness $2 \tau_{1}$ corresponds a critical thickness $2 l_{c}$ of the slab of interstellar matter:

$$
2 l_{c}=\frac{2}{2-3 \varpi} \frac{1}{n_{0} \sigma}\left[1-\frac{3 \varpi}{2} \frac{\sqrt{3}+1}{p(\sqrt{3}+p)}\right] .
$$

The condition of spontaneous collapse of a slab of interstellar matter in the presence of a radiation field coming from both sides is evidently

$$
2 l>2 l_{c} \text {. }
$$

To get a more precise idea of the meaning of condition (13) we now calculate $\varpi$. If we neglect interlocking terms, we can suppose that $\varpi$ is given by the relation

$$
\varpi=\frac{\beta e^{\beta} E_{i}(-\beta)}{\sum_{n} \frac{\beta}{n^{3}} \exp \left(\beta / n^{2}\right) E_{i}\left(-\beta / n^{2}\right)} .
$$

with

$$
\beta=15800,{ }^{\prime} T \text {. }
$$

$\varpi$ and $p$ take the values shown in Table $I$.

We derive from Eq. (11) the following value for $\tau_{1}$ : for

for

$$
\varpi=0, \quad \tau_{1}=0.5 \ln \left(N / n_{0}\right)
$$

$$
\varpi=0.39, \quad \tau_{1}=0.68+0.5 \ln \left(N / n_{0}\right) .
$$

Finally, we derive the values of the critical thickness $l_{c}$ and of the mass of a cube of side $2 l_{c}$; we introduce the two constants $\lambda$ and $\mu$ :

$$
l_{c}=\lambda N^{-2} W 10^{14} ; \quad \frac{M}{M_{\odot}}=\mu\left(10^{14} W\right)^{3} N^{-5}
$$

$\lambda$ and $\mu$ are given by Table II.

For $W=10^{-14}, \varpi=0.39$, the critical thickness $l_{c}$ is 0.4 pc for $N=50$; the corresponding mass is about $M_{\odot}$. Since the mass increase as $N^{-5}$, it is readily seen that usually the masses of ionized hydrogen will be too small for spontaneous collapse; on the other hand, dense clouds of ionized hydrogen will be unstable in the presence of an ionizing radiation field and will spontaneously collapse. The collapse of an HII cloud will ultimately lead to the formation of a cloud of neutral hydrogen, as a consequence of the effect of the various cooling agents contained in interstellar matter. 


\section{DISCUSSION}

BerTRAM Donn, Wayne State University, Detroit, Michigan: Schatzman remarked that in HI regions the formation of CI would tend to eliminate the cooling or prevent condensation. However, if we look at the complete chemical composition of interstellar matter, one has to consider not only the atoms but also the existence of molecules of various degrees of complexity and the grains. Platt has suggested that one can account for the general properties of the obscuration and reddening in terms, not of micron-sized grains, but of large radicals of size about 20 to 50 Angstrom units. These can have appreciable continuous absorption through quantum mechanical effects rather than the ordinary classical scattering. If this hypothesis is true, we have many more particles of molecular type and, between atoms and particles of the order of a hundred or so atoms, there will be a large array of smaller molecules.

If we take the case of an $\mathrm{HI}$ region that starts to condense, then one would expect that the molecular concentration would increase even more. These "molecules" should act as radiators and contribute to the cooling process. I think this is a point that has to be taken more clearly into account when considering the temperature and the condensation of interstellar matter.

E. Schatzman, Institut d'Astrophysique, Paris, France: I do not think I said that interstellar matter would be prevented from cooling. What I said is that the conditions would be different if there would be a CI region, and I said it is very unlikely that there would be any CI region. But another point is that, if the molecules are so efficient, then we must need some other heating agent to keep the interstellar matter at its observed high temperature.

E. N. PARKER, Enrico Fermi Institute for Nuclear Studies, Chicago, Illinois: You mentioned the possibility of starting with a cloud at, say, $1000^{\circ} \mathrm{K}$, and as the outsides of it expand into space, the inner parts possibly might collapse and form some dense cloud. Then you remarked that it was a nonlinear hydrodynamic problem and, therefore, not immediately solvable. Just how tough a problem is it? The reason I ask that question is that I think it is an extremely important point. I was worried about not being able to hold the clouds together that one sees, and this phenomenon might take care of that.

E. SCHATZMAN : As an order of magnitude, we could write that the rate of cooling, which is something like $1 / \gamma n_{\mathrm{H}}$, is of the order of the ratio of the size of the cloud divided by the sound velocity. And that would give the critical size : $l \cong v / \gamma n_{\mathrm{H}}$.

E. A. SPIEGEL, University of Michigan Observatory, Ann Arbor, Michigan: It would seem that the sort of HII region whose stability is studied could only be formed by the radiative interactions which would promote stability. In that case, unstable situations would be unlikely to arise.

E. SchatzMan : I quite agree that there is the question of evolution, how the matter has become ionized, and how later on it became unionized. My purpose was not to treat the full history of the interstellar matter, but to see whether in an HII region there might be new phenomena which would be due to interaction between the optical and the hydrodynamical phenomena. It turned out that such kind of interaction was possible. So that even if the treatment is not quite correct, I think that it should be stressed that one cannot neglect the connection between hydrodynamic phenomena and optical phenomena in the treatment of interstellar matter. 